\title{
Clinical features and prognostic factors for patients with bone metastases from prostate cancer
}

\author{
Jian He, Zhao-Chong Zeng, Ping Yang, Bing Chen, We Jiang and Shi-Suo Du \\ To identify the clinical features and independent predictors of survival in patients with bone metastases from prostate cancer (PCa). We \\ retrospectively analysed $115 \mathrm{PCa}$ patients with bone metastases between 1997 and 2009 . The overall survival rate after bone \\ metastases was calculated using the Kaplan-Meier method. The prognostic factors were identified by univariate analysis using a \\ log-rank test and by multivariate analysis using Cox proportional hazards regression models. The follow-up rate was $100 \%$, the \\ follow-up cases during 1,3 and 5 years were 103,79 and 55 , respectively. The $1-, 3$ - and 5 -year survival rates were $89.1 \%, 60.9 \%$ and \\ $49.8 \%$, respectively, with a median survival time of 48.5 months for patients with bone metastases from PCa. In univariate analysis, \\ age, Gleason score, clinical stage, the number of bone lesions, alkaline phosphatase (ALP) level, invasion of neighbouring organs and \\ non-regional lymph node metastases were correlated with prognosis. By multivariate analysis using Cox regression, ALP level, Gleason \\ score and non-regional lymph node metastases were independent prognostic factors. These prognostic factors will help us to determine \\ the appropriate dose and fraction of radiotherapy for these patients.
}

Asian Journal of Andrology (2012) 14, 505-508; doi:10.1038/aja.2012.24; published online 16 April 2012

Keywords: bone metastases; clinical features; prognostic analysis; prostate cancer

\section{INTRODUCTION}

Prostate cancer (PCa), the most common carcinoma in the industrialised world, has had increasing incidence in China. It is the only solid tumour diagnosed with its first recurrence as bone metastases instead of visceral metastases. ${ }^{1}$ The bones are among the most frequent distant metastatic sites for PCa, occurring in $85 \%-100 \%$ patients. ${ }^{2}$ Some studies $^{3-5}$ have been undertaken to identify prognostic factors for $\mathrm{PCa}$, but less research has been undertaken on bone metastases regularity, especially on the Chinese mainland.

In this retrospective study, 115 cases of PCa with bone metastases were collected, and the clinical features and main prognostic factors, including age, Gleason score, stage, endocrine therapy, castration, radiotherapy, number of bone lesions, prostate-specific antigen (PSA) and alkaline phosphatase (ALP) levels, neighbouring organ metastases, regional and remote lymph node metastases, surrounding parenchyma invaded and other visceral metastases were analysed to determine treatment strategies and to predict the reactions of the patients to treatment.

\section{MATERIALS AND METHODS}

\section{General clinical data}

A total of 115 patients with bone metastases from PCa between 1997 and 2009 were collected from Zhongshan Hospital, with a median age of 72 years (52-91 years). All of the patients were confirmed as having primary PCa by pathological diagnosis and were diagnosed with bone metastases through radioisotope scanning or magnetic resonance imaging. Metastases spread to the spine (43.3\%), in which the lumbar spine $(20.3 \%)$ and the pelvis (19.9\%) were the most frequently involved sites. Figure 1 shows that the most common bone metastatic sites occurred in the bottom of the axis in this study, which was consistent with the PCa blood metastases pathway in the patients' anatomy. ${ }^{6,7}$

\section{Collection of clinicopathological data}

Patient age at diagnosis, Gleason score and clinical stage at initial diagnosis, treatment for primary prostate lesions, including castration, radiotherapy and incretin therapy, number of bone lesions, PSA and ALP levels, prediagnosis of bone cancer, neighbouring organ metastases, regional and remote lymph node metastases, surrounding parenchymal invasion and other visceral metastases, as well as followup duration and survival status, were retrospectively collected and reviewed. Tumour staging was performed according to the 1997 Fifth American Joint Committee Cancer-International Union Against Cancer system. Serum PSA values were determined in the same laboratory using the ELISA method. The normal range was $0-4 \mathrm{ng} \mathrm{ml}^{-1}$. The PSA values of the patients were characterised as $0-4 \mathrm{ng} \mathrm{ml}^{-1}$, $4-20 \mathrm{ng} \mathrm{ml}^{-1}$ or more than $20 \mathrm{ng} \mathrm{ml}^{-1}$. In the pathological examinations of the patients, Gleason scores were characterised as $2-4,5-7$ or $8-10$. When determining the number of metastases by radioisotope scanning, the number of metastases in each vertebra and rib was calculated as 1 ; in the statistical evaluation, the number of bone metastases was assessed as either single or multiple. The study design was approved by the institutional ethics review board of Zhongshan Hospital of Fudan University. 


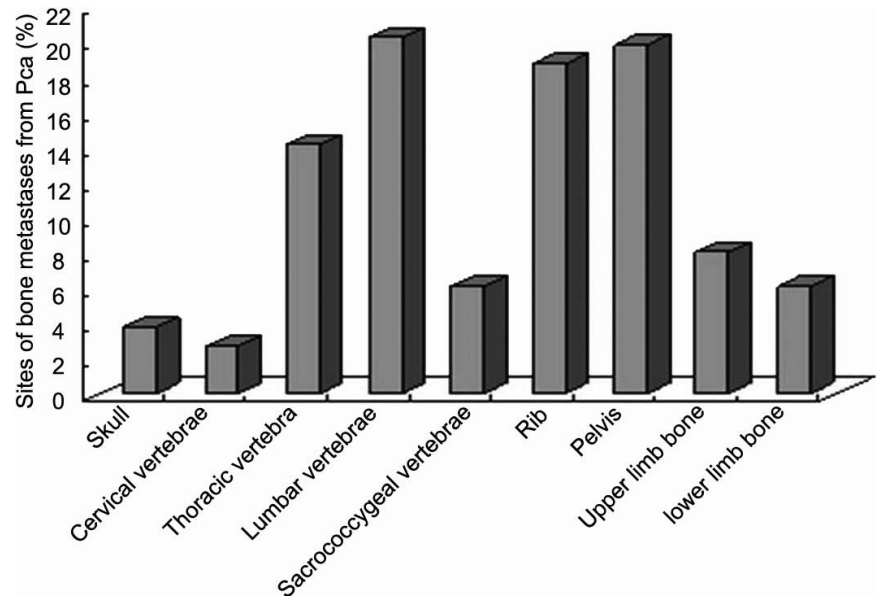

Figure 1 Sites of bone metastases from prostate cancer. PCa, prostate cancer.

\section{Follow-up and statistical methods}

The survival time was defined as from the date the first diagnosis of bone metastases to the date of death or of the last follow-up. The univariate and multivariate analyses were performed using SPSS 15.0 software (IBM, Armonk, NY, USA). The Kaplan-Meier method with a log-rank test was used for survival rate calculations and to evaluate each variable. Multivariate analysis was carried out with the Cox regression model, and all of the variables were entered in a single step, using backward stepwise regression (likelihood ratio). All of the tests were two-sided, and $P<0.05$ was considered statistically significant.

\section{RESULTS}

\section{Patient demographics}

The ages of the patients in this study were between 52 and 91 years (mean: 72 years). A total of 108 patients received treatment, including orchiectomy and/or prostatectomy, for primary tumours of the prostate, and 100 patients received endocrine therapy. When bone metastases occurred, most of the patients $(64.3 \%)$ had increased levels of PSA (>20 ng ml$\left.{ }^{-1}\right)$.

\section{Survival time analysis}

All of the patients (100\%) were followed up until the date of death or 31 December 2009. The median follow-up was 26.8 months (interquartile range: 6.0-76.5 months). The follow-up cases during 1, 3 and 5 years were 103, 79 and 55, respectively. The 1-, 2-, 3- and 5-year survival rates were $89.1 \%, 76.9 \%, 60.9 \%$ and $49.8 \%$, respectively, with a median survival time of 48.5 months (0.5-74.5 months) from the diagnosis of bone metastases, while the 1-, 2-, 3- and 5-year survival rates were $91.8 \%, 80.1 \%, 64.0 \%$ and $46.7 \%$, respectively, with a median survival time of 58.5 months (1-223 months) from a diagnosis of the PCa.

\section{Prognostic factors in univariate and multivariate analysis}

The results of univariate analysis showed that survival was associated with the patient's age, Gleason score and clinical stage at the diagnosis of the primary tumour, the number of bone metastases, ALP, adjacent organ metastases and non-regional lymph node metastases $(P<0.05)$. The other factors were not statistically significant $(P>0.05$, Table 1$)$.

Multivariate Cox regression analysis showed that ALP levels, Gleason score at diagnosis of the primary tumour and non-regional lymph node metastases were statistically significant factors for overall survival from the diagnosis of bone metastases (Table 2).

\section{DISCUSSION}

Although the early diagnostic methods for PCa are advanced (e.g., PSA screening, high-resolution magnetic resonance imaging, ultrasonic testing), approximately $10 \%-20 \%$ of PCa patients are initially diagnosed because of bone metastases. ${ }^{8}$ After castration therapy or hormone therapy for 18-36 months, many patients appear androgenindependent, and 90\%-95\% patients suffer from bone metastases. Of patients who died of $\mathrm{PCa}$, the incidence of bone metastases at the autopsy examination was $65 \%-85 \% .^{9}$ Bone metastases of PCa often occurs in rich blood supply sites, such as the vertebrae, pelvic bones, ribs, skull and proximal long bones, as well as other parts. ${ }^{10}$ The low lumbar spine is the most common site of bone metastases. ${ }^{11}$ Some scholars believe that the bones are given priority with respect to blood circulation from the prostate, resulting in a high probability of cancer cells metastasising to the bones. We have observed that bone metastases from PCa occurred mainly in the spine and pelvis, with the lumbar spine the most frequent site, which is consistent with some reports. $^{12,13}$

The patients in our study ranged in age from 52 to 91 years (mean: 72 years), showing that bone metastases of PCa was more common in older males with multiple metastases and mostly without extension into the adjacent soft tissues and organs. PCa does not usually metastases to the lymph nodes or other organs.

The effect of age on survival was statistically insignificant in our study; however, some researchers have reported that younger men with high-risk disease appear to have poorer prognoses than older men with PCa. ${ }^{14}$ Thus, selectivity might exist in retrospective studies. The number of bone lesions was reported to be a prognostic factor. ${ }^{15}$ In this study, patients with multiple bone metastases had statistically poorer borderline survival; a prospective study might provide the precise role of this factor in these patients.

ALP levels at the diagnosis of bone metastases, Gleason score at the diagnosis of the primary tumour and non-regional lymph node metastases were found to be independent prognostic factors. Serum ALP level has been found to be an important prognostic factor in many studies. ${ }^{15-17}$ Our results agree with the data from the literature.

The median overall survival of patients with Gleason scores $\geqslant 8$ was significantly longer than that of the lower-score patients. Cheville et al. ${ }^{18}$ and Yigitbasi et al. ${ }^{15}$ also indicated that among patients with bone metastases, the Gleason score of the primary prostatic adenocarcinoma was associated strongly with patient outcome.

PSA is important in the diagnosing, staging and follow-up of $\mathrm{PCa}$, and Goya et al. ${ }^{19}$ suggested that PSA plays a crucial role in osteoblastic bone metastases by promoting both osteoblast proliferation and apoptosis of osteoclast precursors. However, regarding PSA as a prognostic factor, there exist different opinions in the literature, especially for bone metastatic patients. Glass et al. $^{20}$ stated that PSA was an independent predictor of metastatic PCa, but other researchers have reported that pretreatment PSA level did not have a statistically significant effect on survival in patients with bone metastases..$^{15,21,22}$ In our study, PSA was also identified as having an insignificant effect on survival. Regarding such cases, Sasaki et al. ${ }^{23}$ provided a promising opinion that nadir PSA levels and the time to PSA nadir following primary androgen deprivation therapy might be the most important early predictors for longer survival in PCa patients with bone metastases. 
Table 1 Univariate analysis for survival of 115 patients with bone metastases from prostate cancer

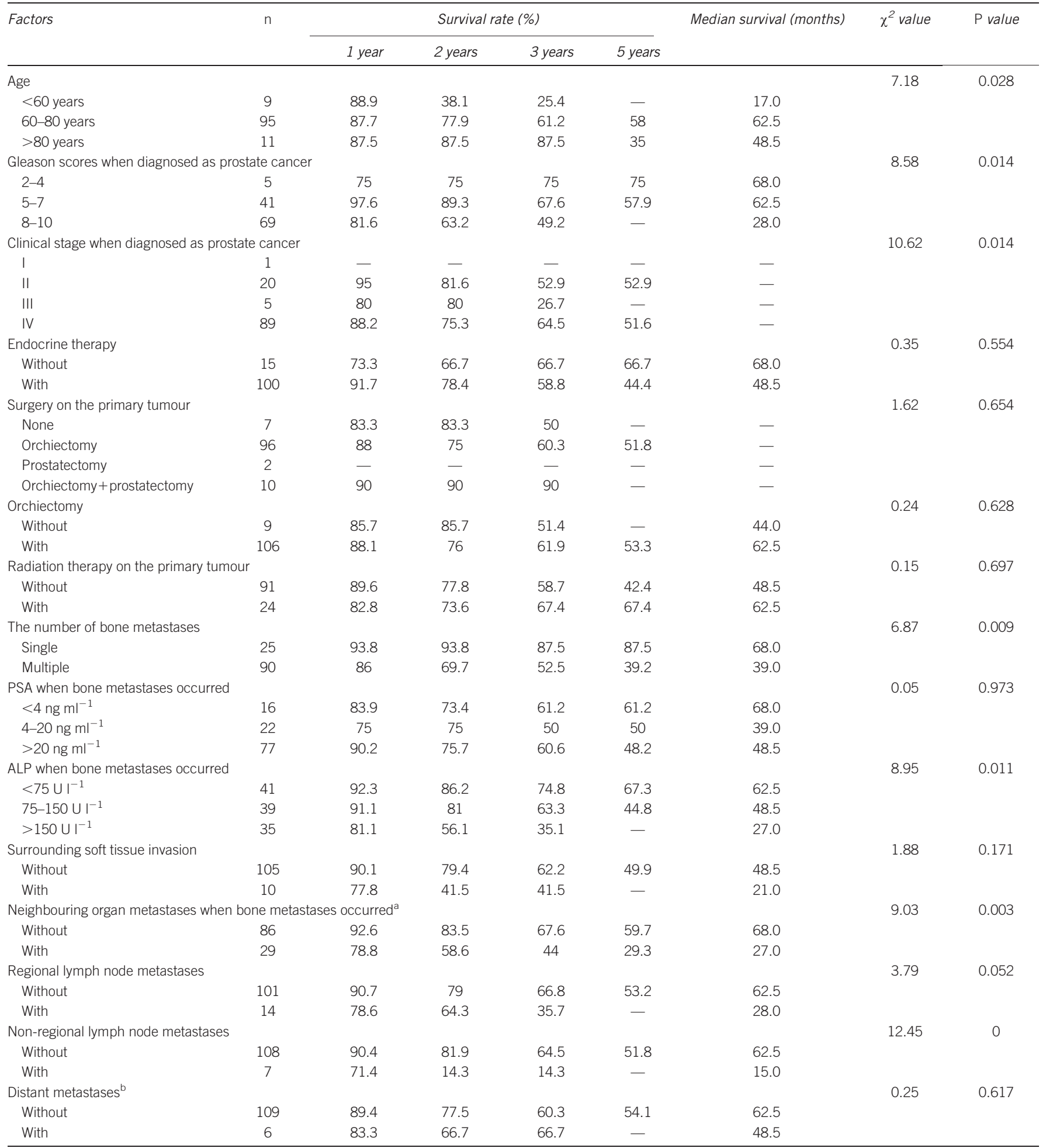

Abbreviations: ALP, alkaline phosphatase; PSA, prostate-specific antigen.

${ }^{a}$ Defined as an organ around with prostate, such as the bladder, spermatophores, urethra and rectum.

${ }^{\mathrm{b}}$ Defined as brain, lung and liver metastases.

- censored data. 
Table 2 Multivariate analysis for survival of 115 patients with bone metastases from prostate cancer

\begin{tabular}{lcccccc}
\hline Factors & $\beta$ & s.e. & $R R$ & $R R(95 \%$ Cl) & $\chi^{2}$ & $P$ \\
\hline Number of bone metastases \\
1.296 & 0.753 & 3.656 & $0.836-15.991$ & 4.099 & 0.085 \\
\multicolumn{7}{c}{ ALP levels when bone metastases occurred } \\
0.001 & 0.000 & 1.001 & $1.001-1.002$ & 4.077 & 0.020 \\
Glasson score when bone metastases occurred \\
0.715 & 0.354 & 2.044 & $1.021-4.094$ & 4.573 & 0.044 \\
Age & -0.703 & 0.379 & 0.495 & $0.235-1.041$ & 3.353 & 0.064 \\
Non-regional lymph node metastases \\
1.05 & 0.483 & 2.856 & $1.109-7.357$ & 3.934 & 0.030 \\
\hline
\end{tabular}

Abbreviations: ALP, alkaline phosphatase; $\mathrm{Cl}$, confidence interval; RR, relative risk; s.e., standard error.

$\beta$ : coefficient of regression.

Saeter $e$ al. ${ }^{24}$ found that the survival of patients with soft tissue or non-regional lymph node metastases was similar to those patients presenting with skeletal metastases only, but few studies have discussed the prognostic value of these factors. The present study was the first to find that non-regional lymph node metastases might be an independent predictor in metastatic PCa patients.

The patient survival analysis showed that the 5-year survival rate starting from the diagnosis of PCa was lower than that starting from the diagnosis of bone metastases. This 'inversion' phenomenon can be explained by bone metastases often being the first clinical symptom in $\mathrm{PCa}^{25}$

In our patient cohort, the prognoses were better than those in previous reports. ${ }^{22}$ The reason for this finding might be the use of comprehensive therapy in clinical practice, including radiotherapy and endocrine therapy, among others. These therapies prolong survival time and improve quality of life. Bone metastases is a dangerous clinical problem for advanced PCa patients, causing pain, pathologic fractures, spinal cord compression, limited mobility, bone marrow suppression, anaemia, thrombocytopaenia and so on; thus, active treatment should be undertaken. Prognostic indicators are of major clinical importance in the treatment of patients with metastatic PCa. For patients with different prognoses, proper individual therapeutic approaches are needed. Radiotherapy is a very effective method. Generally, hyperfractionated radiotherapy has been used to treat patients with short expected survival times to relieve their pain, and conventional fractionated radiotherapy has been administered to patients with longer expected survival times to protect normal tissue. Therefore, the dose of radiation therapy should be considered based on the specific conditions of each patient. As the survival time of patients with bone metastases from PCa is relatively long, conventional fractionated radiotherapy should generally predominate. Patients with weight-bearing bone metastases should be treated with higher doses of radiotherapy to enhance autonomous activity and preserve quality of life.

\section{CONCLUSIONS}

In conclusion, long-term survival in patients with bone metastases from PCa is higher than with bone metastases from other cancers. ALP level, Gleason score and non-regional lymph node metastases were independent predictors of patients with bone metastases from PCa. These data can serve as a guide for clinical management and for determining the prognosis of PCa with bone metastases.

\section{AUTHOR CONTRIBUTIONS}

$\mathrm{JH}$ designed the study, collected, analyzed and interpreted the clinical data and wrote the manuscript. ZCZ designed the study, supervised the project and revised the manuscript. PY, BC and SSD collected partial patients' clinical data. WJ revised the manuscript. All authors vouch for the respective data and analysis, approved the final version and agreed to publish the manuscript.

\section{COMPETING FINANCIAL INTERESTS}

For all authors, no potential conflicts of interest exist.

1 Dallari R, Gazzotti GL, Barozzini P, Cervi F, Cavani R. [Bone metastases as a sign of the existence of latent neoplasia]. Minerva Med 1986; 77: 981-7. Italian.

2 Drago JR. Diagnostic techniques in prostatic cancer. Postgrad Med 1986; 80: 214-7.

3 Buhmeida A, Pyrhönen S, Laato M, Collan Y. Prognostic factors in prostate cancer. Diagn Pathol 2006; 3 : 4.

4 Steuber T, O'Brien MF, Lilja H. Serum markers for prostate cancer: a rational approach to the literature. Eur Urol 2008; 54: 31-40.

5 Shariat SF, Semjonow A, Lilja H, Savage C, Vickers AJ et al. Tumor markers in prostate cancer I: blood-based markers. Acta Oncol 2011; 50 Suppl 1: 61-75.

6 Kageyama Y, Kihara K, Kamata S, Nagahama K, Yonese J et al. [Relationship between pretreatment serum levels of prostate specific antigen and bone metastases in prostate cancer]. Hinyokika Kiyo 1996; 42: 197-9. Japanese.

7 Xu J, Wang R, Xie ZH, Odero-Marah V, Pathak S et al. Prostate cancer metastases: role of the host microenvironment in promoting epithelial to mesenchymal transition and increased bone and adrenal gland metastases. Prostate 2006; 66: 1664-73.

8 Imai K, Suzuki T, Shimizu T, Yamanaka H, Kanetake $\mathrm{H}$ et al. Analysis of survival of prostate cancer patients in Japan and the U.S.A. Adv Exp Med Biol 1992; 324: 29-38.

9 Reddi AH, Roodman D, Freeman C, Mohla S. Mechanisms of tumor metastases to the bone: challenges and opportunities. J Bone Miner Res 2003; 18: 190-4.

10 Conti G, La Torre G, Cicalese V, Micheletti G, Ludovico MG et al. Prostate cancer metastases to bone: observational study for the evaluation of clinical presentation, course and treatment patterns. Presentation of the METAURO protocol and of patient baseline features. Arch Ital Urol Androl 2008; 80: 59-64.

11 Chen SS, Chen KK, Lin AT, Chang YH, Wu HH et al. The significance of serum alkaline phosphatase bone isoenzyme in prostatic carcinoma with bony metastases. $\mathrm{Br} \mathrm{J} \mathrm{Urol}$ 1997; 79: 217-20.

12 Knerr K, Ackermann K, Neidhart T, Pyerin W. Bone metastases: osteoblasts affect growth and adhesion regulons in prostate tumor cells and provoke osteomimicry. Int $\mathrm{J}$ Cancer 2004; 111: 152-9.

13 Bubendorf L, Schopfer A, Wagner U, Sauter G, Moch H et al. Metastatic patterns of prostate cancer: an autopsy study of 1,589 patients. Hum Pathol 2000; 31: 578-3.

14 Abouassaly R, Paciorek A, Ryan CJ, Carroll PR, Klein EA. Predictors of clinical metastases in prostate cancer patients receiving androgen deprivation therapy: results from CaPSURE. Cancer 2009; 115: 4470-6.

15 Yigitbasi O, Ozturk U, Goktug HN, Gucuk A, Bakirtas H. Prognostic factors in metastatic prostate cancer. Urol Oncol 2011; 29: 162-5.

16 de Voogt HJ, Suciu S, Sylvester R, Pavone-Macaluso M, Smith PH et al. Multivariate analysis of prognostic factors in patients with advanced prostatic cancer: results from 2 European Organization for Research on Treatment of Cancer trials. J Urol 1989; 141: 883-8.

17 Jorgensen T, Kanagasingam Y, Kaalhus O, Tveter KJ, Bryne M et al. Prognostic factors in patients with metastatic (stage D2) prostate cancer: experience from the Scandinavian Prostatic Cancer Group Study-2. J Urol 1997; 158: 164-70.

18 Cheville JC, Tindall D, Boelter C, Jenkins R, Lohse CM et al. Metastatic prostate carcinoma to bone: clinical and pathologic features associated with cancer-specific survival. Cancer 2002; 95: 1028-36.

19 Goya M, Ishii G, Miyamoto S, Hasebe T, Nagai K et al. Prostate-specific antigen induces apoptosis of osteoclast precursors: potential role in osteoblastic bone metastases of prostate cancer. Prostate 2006; 66: 1573-84.

20 Glass TR, Tangen CM, Crawford ED, Thompson I. Metastatic carcinoma of the prostate: identifying prognostic groups using recursive partitioning. J Urol 2003; 169: 164-9.

21 Kawai M, Uemura H, Hasumi H, Osada Y, Ohta J et al. [Usefulness of extension of disease as a prognostic factor in relapsed prostate cancer patients of stage D]. Nippon Hinyokika Gakkai Zasshi 2005; 96: 442-7. Japanese.

22 Chen CH, Tzai TS, Huang SP, Wu HC, Tai HC et al. Clinical outcome of Taiwanese men with metastatic prostate cancer compared with other ethnic groups. Urology 2008; 72: 1287-92.

23 Sasaki T, Onishi T, Hoshina A. Nadir PSA level and time to PSA nadir following primary androgen deprivation therapy are the early survival predictors for prostate cancer patients with bone metastases. Prostate Cancer Prostatic Dis 2011; 14: 248-52.

24 Saeter G, Fossa SD, Ous S, Blom GP, Kaalhus O. Carcinoma of the prostate with soft tissue or non-regional lymphatic metastases at the time of diagnosis: a review of 47 cases. Br J Urol 1984; 56: 385-90.

25 El Otmany A, Bouklata S, Hafid H, Chami I, Jalil A et al. [Bone metastases revealing a primary prostate cancer]. J Radiol 2000; 81: 990-1. French. 